\title{
Efficacy of Glucagon-Like Peptide-1 Receptor Agonists in the Weight Loss Among Obese Individuals: A Systematic Review
}

\author{
Raed Aldahash ${ }^{\mathrm{a}, \mathrm{b}, \mathrm{c}} \mathbb{C}$
}

\begin{abstract}
Background: Obesity can seriously damage human health and have the potential to raise the likelihood of diabetes mellitus (DM) and other adverse outcomes. Successful therapeutic options and medications have been designed to reduce weight. Glucagon-like peptide-1 receptor agonists (GLP-1Ras) are recommended to reduce the weight among obese patients either with or without type 2 DM (T2DM). We intended to perform the systematic review to synthesize the findings from the studies that have explored the efficacy of GLP-1Ras in reducing weight among obese individuals.
\end{abstract}

Methods: A wide range of electronic bibliographic databases such as PubMed, Embase, and ERIC was searched. Based on the eligibility criteria, both observational and non-observational (experimental) studies that assessed the efficacy of GLP-1Ras in reducing weight loss among obese individuals from January 2010 to July 2021 were incorporated in the review. Following screening and assessing the abstracts, we ended up reviewing 20 full-text articles, and data were extracted on important parameters such as country, sample size, type of non-surgical treatment, time of follow-up, and primary outcomes.

Results: Overall, the findings of the systematic review appear promising for the efficacy of different GLP-1Ras in reducing the weight and related parameters of obesity such as body mass index and lean body mass. More precisely, individuals lost weight of about minimum of $5.1 \mathrm{~kg}$ and maximum of $6.16 \mathrm{~kg}$ in the intervention group or those who were observed to use any type of GLP-1Ras as opposed to 1.6 - $3.97 \mathrm{~kg}$ lost among those individuals who did not use any type of GLP-1Ras. These results with their respective effect sizes were statistically significant with a P-value of $<0.05$. A wide variety of GLP-1Ras such as liraglutide, exenatide, semaglutide, and dulaglutide are considered safe to reduce weight loss among individuals aged

Manuscript submitted April 28, 2021, accepted August 9, 2021

Published online October 31, 2021

${ }^{a}$ Department of Medicine, Ministry of National Guard - Health Affairs, Riyadh, Saudi Arabia

${ }^{b}$ King Abdullah International Medical Research Center (KAIMRC), Riyadh, Saudi Arabia

${ }^{\mathrm{c}}$ King Saud bin Abdulaziz for Health Science, Riyadh, Saudi Arabia.

Email: dr.raaed@hotmail.com

doi: https://doi.org/10.14740/jem745
18 - 65 years. Out of 13 studies included in this review, 12 showed statistically significant results with a P-value of less than 0.05 in all the included studies.

Conclusion: Given their likely advantages further than glycemic control in reducing the weight, GLP-1 agonists may help to treat the obesity either among diabetic or non-diabetic individuals soon. Though, further research studies mainly large clinical trials are required to broaden and completely explain the favorable effects and potential side effects of GLP-1 agonists.

Keywords: Glucagon-like peptide-1 receptor agonists; Obesity; Weight loss; Systematic review

\section{Introduction}

Obesity and overweight can seriously damage human health and have the potential to raise the likelihood of type 2 diabetes mellitus (T2DM) and other adverse outcomes including resistance to insulin at the cellular level, hyperlipidemia, and heart ailments $[1,2]$. According to the World Health Organization, around 1.5 billion adults were labeled as overweight in 2011 and around 2.8 million deaths occur among adults annually that are attributed to overweight or obesity [3]. More than $80 \%$ of patients with T2DM suffer from obesity or overweight and around three-fourths of patients with DM might experience complications such as diseases of the vessels and other DMassociated complications due to obesity $[4,5]$. Many clinicians and also patients strive to lose weight or weight gain while controlling the glucose levels of patients. Both doctors and patients aim to reduce weight and reduce the adverse effects of T2DM or manage their glucose levels [4].

Successful therapeutic options and medications both pharmacological and non-pharmacological interventions have been designed to reduce the weight mainly among patients with DM to reduce the risk of a myriad of impediments [6,7]. However, it has been found that medications such as sulphonylureas, insulin, and thioglitazones that are used to manage DM increase weight. On the other hand, metformin, dipeptidyl peptidase 4 inhibitors (DPP4is), sodium-glucose cotransporter-2 (SGLT-2) inhibitors and glucagon-like peptide receptor agonists (GLP$1 \mathrm{RAs})$ reduce weight along with appropriate glycemic control. 
Therefore, there is a tendency to choose the options that manage glycemic control with no increments in the weight. One of such options is glucagon-like peptide-1 receptor agonists (GLP-1Ras), which are recommended to reduce the weight among obese patients either with or without T2DM. More specifically, GLP-1Ras is a hormone that is discharged from the gut (intestine) after an individual eats a meal, which in turn triggers the production of insulin and prevents the release of glucagon [8]. This hormone can suppress the appetite and slow down the gastric and stimulate satiation, thereby it plays an essential role to regulate blood glucose and reduce weight among obese individuals [9]. Furthermore, GLP-1Ras could promote satiation by attaching to its receptor on neurons in the hypothalamus and decrease caloric consumption by delaying gastric emptying $[10,11]$. There is evidence that by the above mechanisms, GLP-1Ras have the potential to reduce the weight of patients either with or without DM. However, there is a need to review and synthesize the findings of both observational and experimental studies to explore the role of GLP1 Ras in reducing weight and the extent to which these GLP1 Ras can reduce weight. Therefore, we intended to conduct a systematic review to synthesize the findings from the studies that have explored the efficacy of GLP-1Ras on reducing the weight among obese individuals.

\section{Materials and Methods}

We performed a review systematically to evaluate, synthesize, and combine the existing evidence on the findings regarding the effect of GLP-1Ras on weight reduction. We used PRISMA guidelines to undertake this systematic review as shown in Table 1 [12].

\section{Inclusion and exclusion criteria}

To answer the study question, the eligibility of a study was contingent for inclusion if a research study evaluated the effectiveness or efficacy of GLP-1Ras to manage obesity, published in English from 2010 to 2020 across different regions of the world. Additionally, only those studies which were quantitative were incorporated. Qualitative studies were excluded and studies without full texts were also excluded. All those studies that consisted of opinions, criticisms of older research studies, and editorials were not included rather studies that compared the efficacy, safety, and effectiveness of GLP-1Ras and their full texts were scrutinized.

\section{Sources of information and strategy for searching the rel- evant articles}

A systematic search of published articles was started and completed in 2021. We searched databases including PubMed, Embase, and ERIC such as Medline, Ovid, and EBSCO. We explored references of pertinent reviews along with the database searches. An independent search was carried out by two authors who also scanned the results for potentially appropriate studies followed by retrieving the full-text articles. The primary endpoint of the review was the efficacy and safety of GLP-1Ras in reducing the weight among obese individuals, which reflected an improvement in the body mass index (BMI) or weight, and lean body mass. We pre-piloted the search strategies without any restrictions by year of publication, geographic area, or other socio-demographic characteristics.

We identified a blend of Medical Subject Heading (MeSH) keywords and text words. We clustered these into four major groups based on the categories of population, intervention, comparison, and outcome as shown in Table 2. The most prevalent search terms found in abstracts and titles comprised "GLP-1Ras" "glucagon-like peptide-1 receptor agonists (GLP-1Ras)", "liraglutide", and "exenatide". Further, we consulted with a librarian to generate a search in four different parts. The first part was restricted to search terms particular to the primary endpoint such as "efficacy of GLP-1Ras", and the second part was for the terms limited to obesity including "reduction in the weight". Besides, we also considered using diverse wordings of main concepts such as obesity management, weight loss, and its management to obtain pertinent research papers. This was followed by combining these major concepts using combinations (AND, OR) relevant to the research question. Moreover, to detect more research articles, we also used truncation $(*)$ with the same root word. We used truncation to make sure to retrieve all potential variants of search terms. We also applied search limits or filters on the language (English), however, and applied restrictions on publication period, age group, and type of studies to include eligible studies in the search.

\section{Data abstraction}

We imported all appropriate research studies into the reference manager software (EndNote) file, where each study was reviewed, and we also screened titles for duplicates in this software. We did not consider the abstracts for further review, which did not explicitly explore the study objective. Finally, we obtained and examined the full-text articles of the remaining relevant articles. This was followed by abstracting and summarizing the articles that met the eligibility criteria using a standardized proforma. Thus, after the process of removing duplicates, title, and abstract screening, we removed papers that were beyond the scope of this review as guided by inclusion criteria. Besides, the bibliography of the remaining studies was also verified and examined to evade missing any useful studies. This process of searching the articles was carried out independently by the reviewers, and their judgments and extracted summaries were matched to identify the differences and resolve these accordingly.

Independent reviewers filled a standardized data extraction sheet for the eligible research articles. The reviewers compared the data extraction tables to ensure including the imperative findings of the eligible studies and pilot tested the data extraction sheet before starting the process of data extraction. Besides, prevailing research articles on the chosen topic were reviewed to describe objects of the data extraction proforma. 
Table 1. PRISMA Check List That Was Followed for This Review

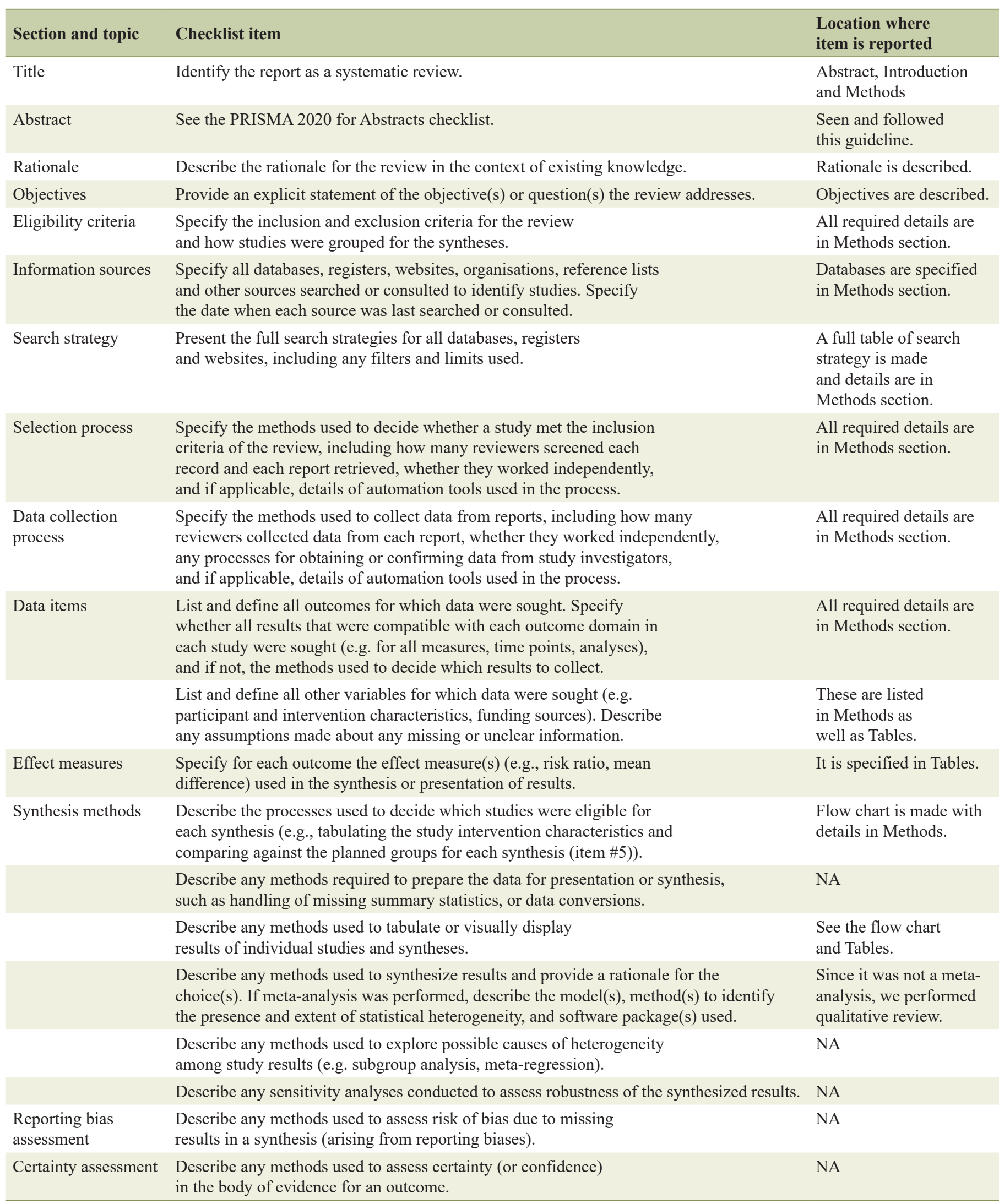


Table 1. PRISMA Check List That Was Followed for This Review - (continued)

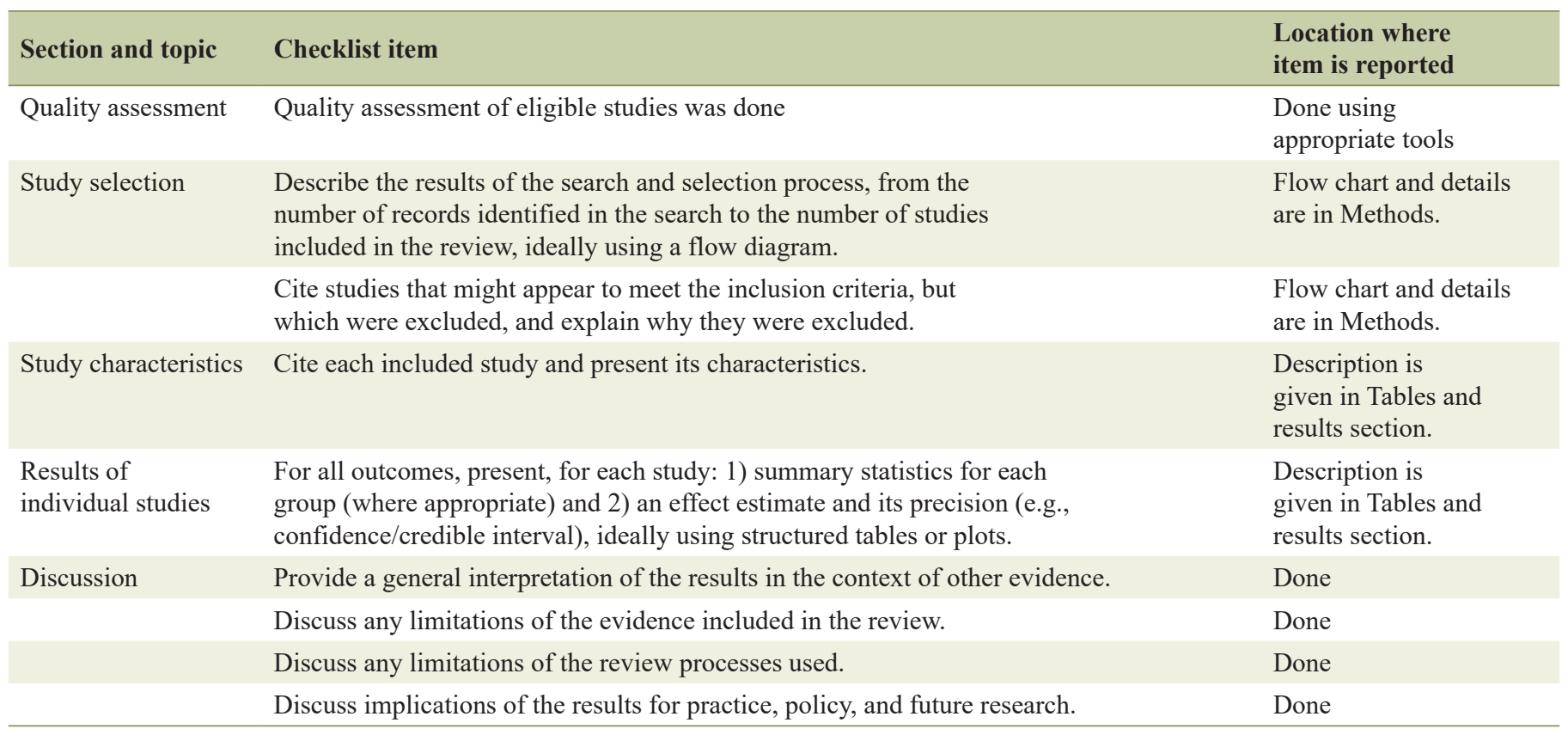

Any discrepancies between the two reviewers were solved by agreement between the two reviewers. The abstracted data comprised of the author, reference, year of publication, type of study design; total study size and population; average age with range for age, randomization group, duration for follow-up, major study findings, and conclusion of the study.

\section{Results}

\section{Findings of the search strategy}

We screened the identified articles initially by titles, then by abstracts, and finally, we carried out a full-text articles assessment. Our initial search identified 1,209 citations in different databases; however, 703 articles were duplicates that were removed. Of the remaining 506 unique studies, we reviewed titles and abstracts and found 96 relevant abstracts. Upon re- viewing abstracts, 69 articles did not meet the eligibility criteria while reviewing the abstracts and seven did not meet eligibility after reviewing full texts. Hence, we were able to retrieve full texts for 20 articles, which were incorporated in the review as shown in Figure 1.

\section{Characteristics of the eligible studies}

With respect to the study design, five of the studies were prospective case series, seven were randomized controlled trials (RCTs), and one was a cohort study. The sample size of all included research studies varied between 9 and 564 with an equal distribution between patients who were and were not randomized to different treatment modalities under the umbrella of GLP-1 agonists in the RCTs. The studies were conducted mostly in developed countries such as USA $(n=4)$, Japan $(n=1$, Europe $(n=1)$, Italy $(n=2)$, China $(n=2)$, Korea $(\mathrm{n}=1)$, UK $(\mathrm{n}=1)$, and Denmark $(\mathrm{n}=1)$ (Table 3 [13-32]).

Table 2. Search Strategy According to PICO Criteria

\begin{tabular}{ll}
\hline Population & "Adults*" [Mesh] OR "women*adults*" OR obese*men or women OR type-2 diabetes mellitus* OR overweight* OR "diabetic*" \\
& OR "adults with type-2 diabetes mellitus" OR "obese adults" OR "diabetic women" "diabetic men" OR "obese women" "obese \\
& men" [Mesh]
\end{tabular}



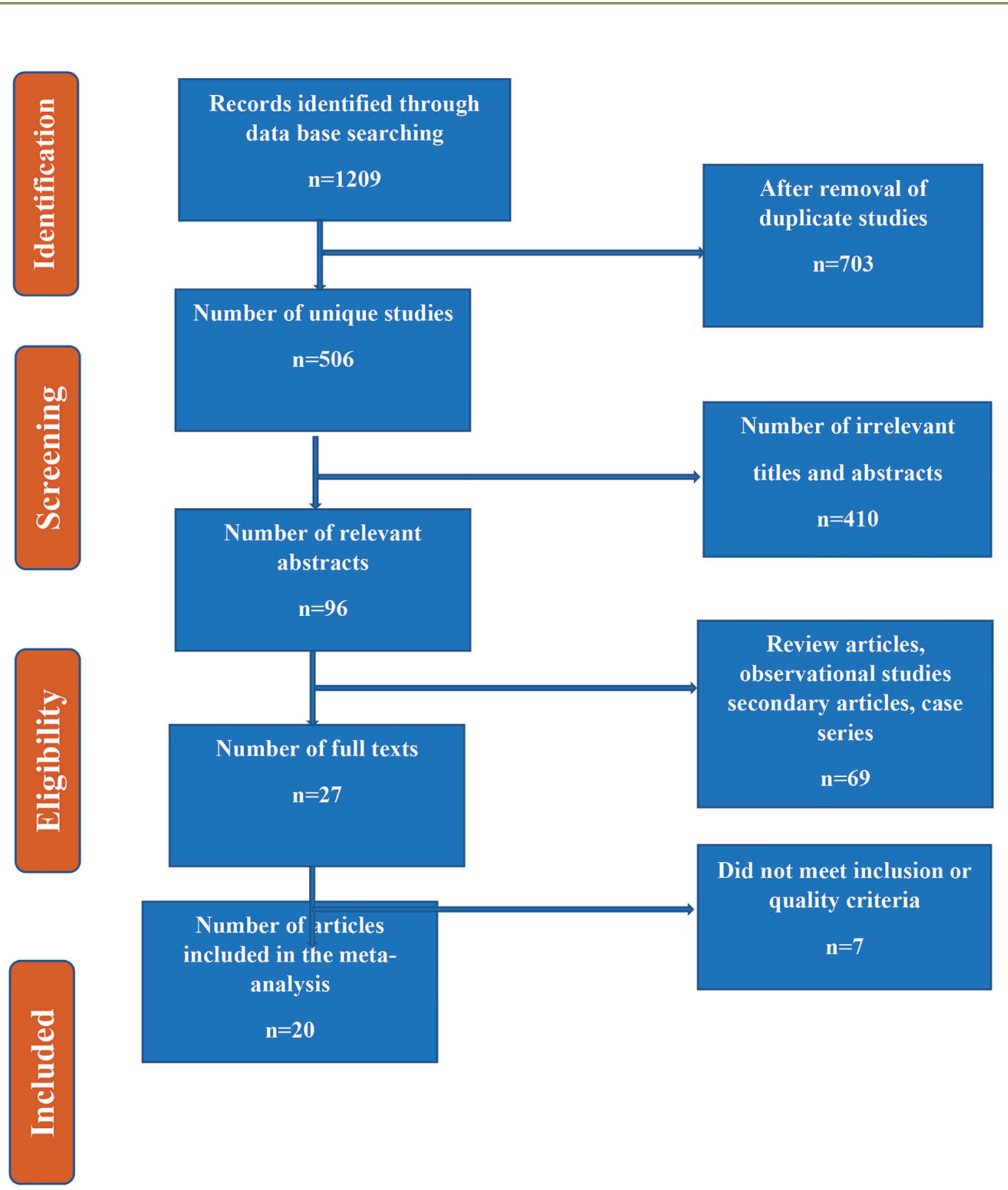


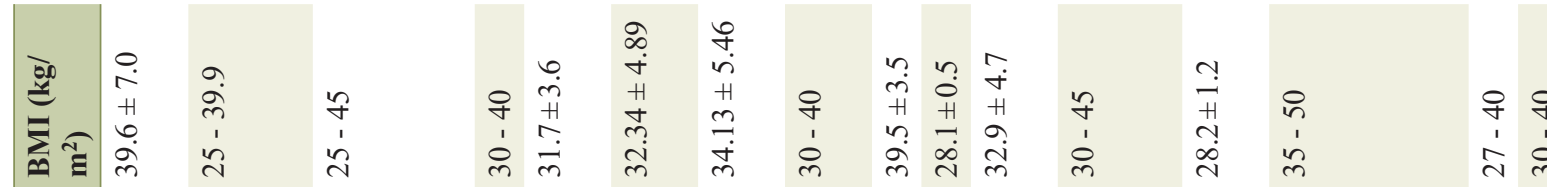

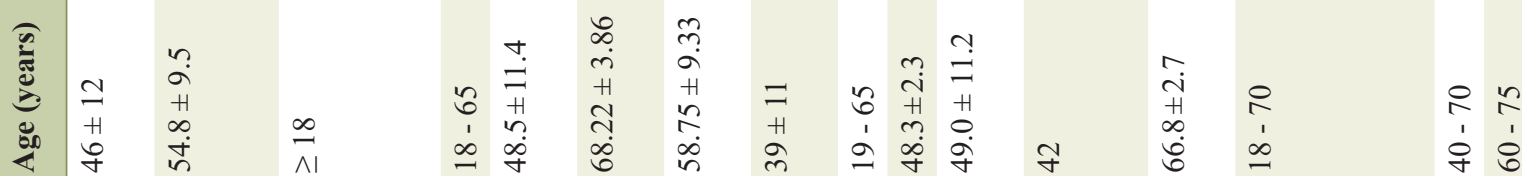

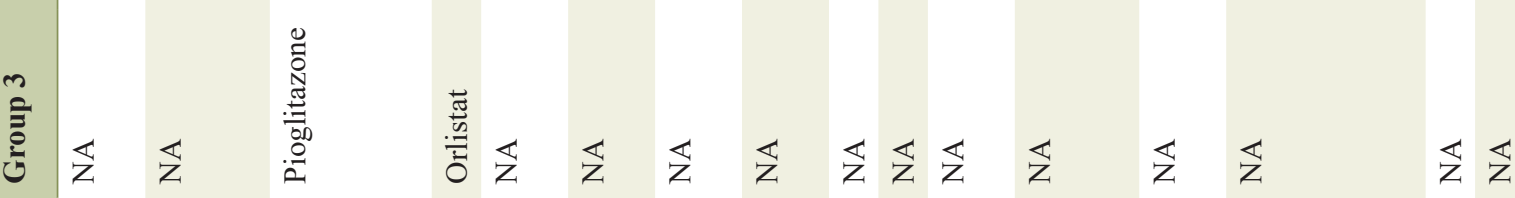

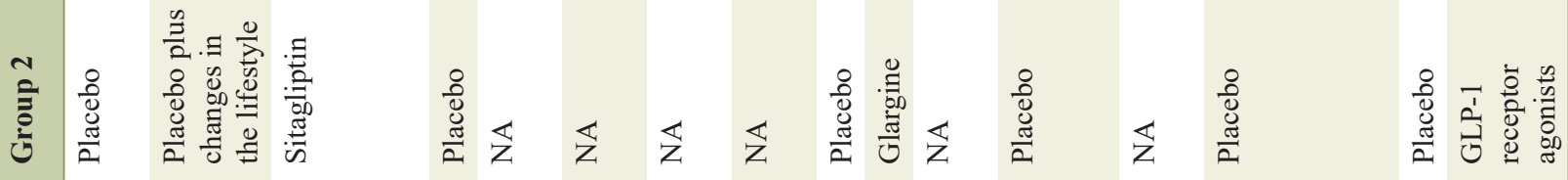

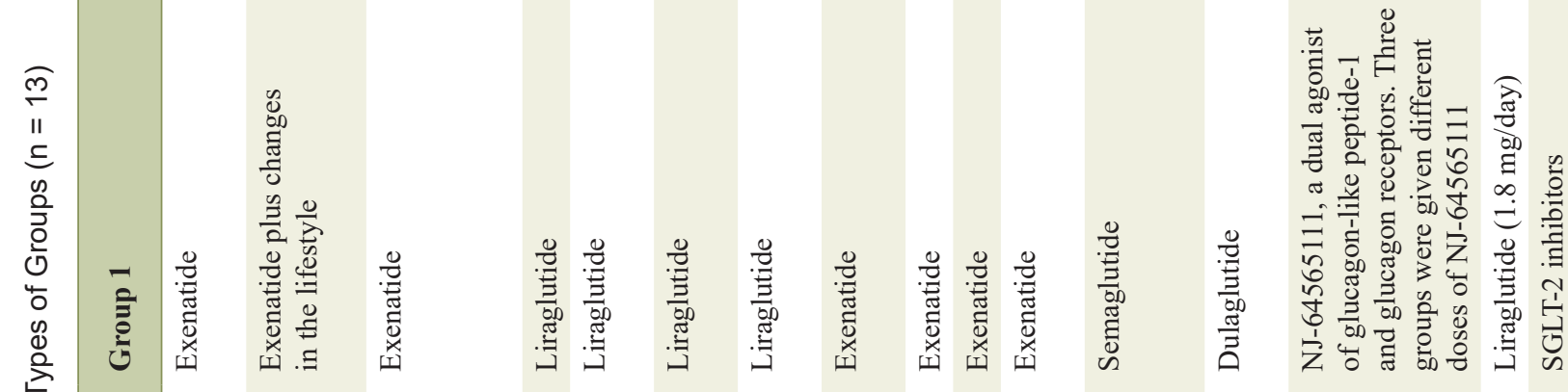

कृ

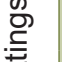

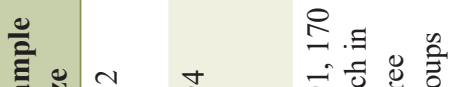

जू

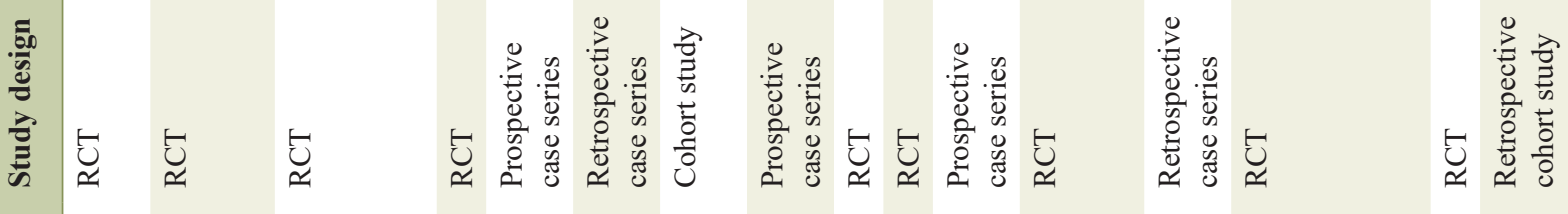

o

索

$\frac{0}{\frac{9}{9}}$

党

$$
\text { 旁 }
$$

赔

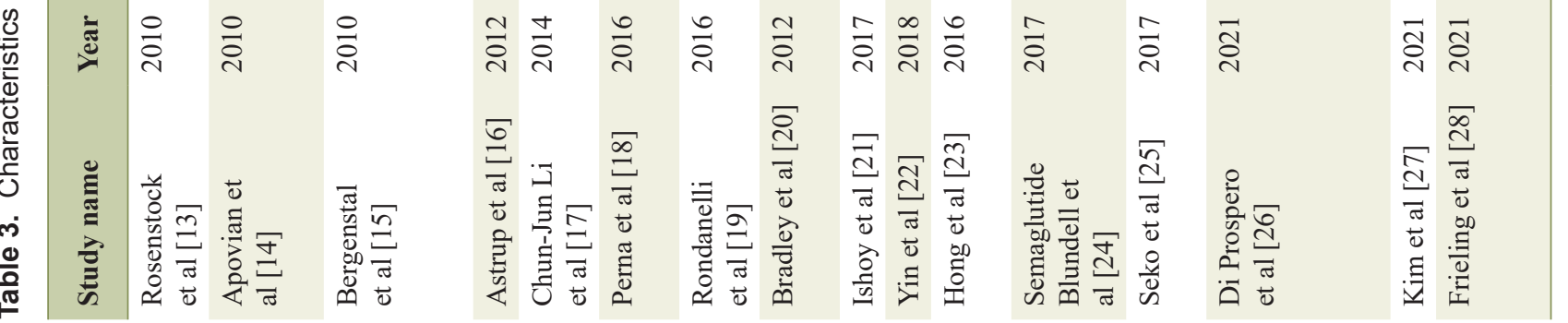




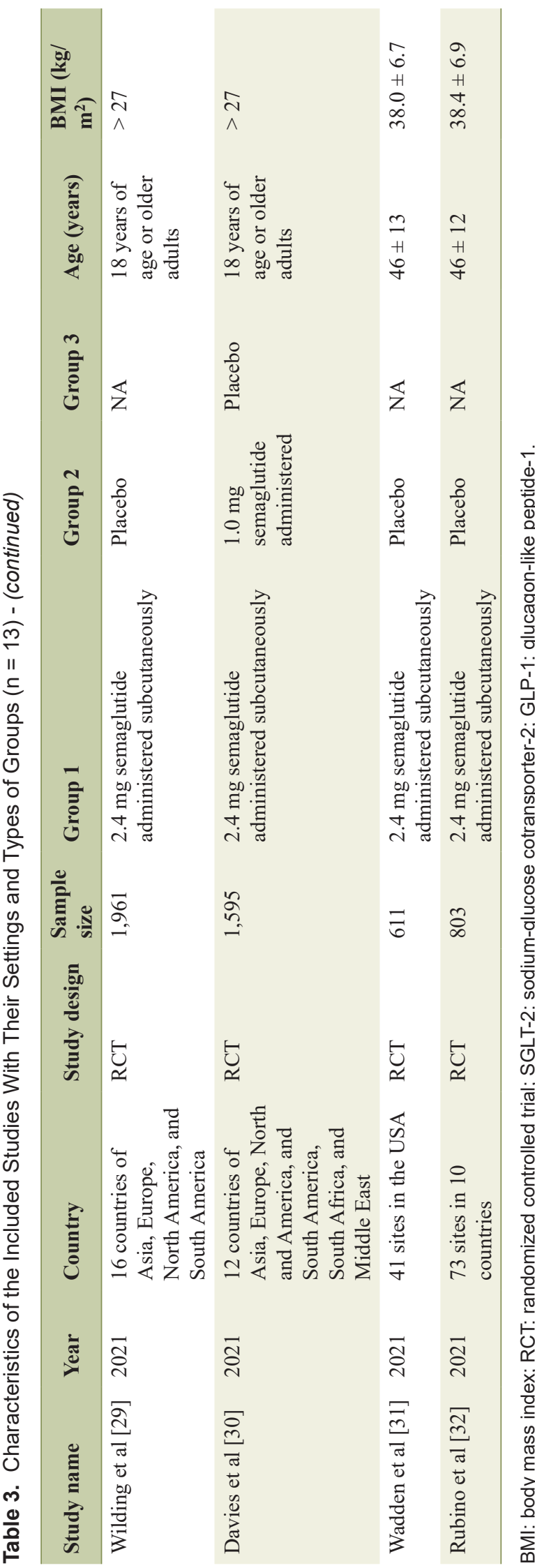

agonists on weight loss.

Overall, most of the studies found favorable results regarding the effect of GLP-1 agonists on the loss of weight as there was a decrease in weight from baseline till follow-up in almost all of the RCTs. For example, one of the studies conducted by Rosenstock et al in 2010 on obese individuals followed study participants for 24 weeks after randomizing one group to GLP1 agonist [13]. The authors found that study participants in the intervention group reduced $5.1 \pm 0.5 \mathrm{~kg}$ when compared to baseline as opposed to $1.6 \pm 0.5 \mathrm{~kg}$ in the control arm (P < $0.001)$. They concluded that exenatide along with the lifestyle changes resulted in a decrease in caloric intake, thereby leading to weight loss among obese individuals and also resulted in improved tolerance to glucose [13]. Likewise, another study conducted by Apovian et al in the same year randomized obese and diabetic individuals to exenatide along with the changes in lifestyle or placebo and followed them for 24 weeks [14]. The study findings revealed that exenatide along with the changes in lifestyle revealed a bigger difference in weight $(-6.16 \pm 0.54$ $\mathrm{kg}$ as opposed to the placebo group $3.97 \pm 0.52 \mathrm{~kg}, \mathrm{P}=0.003)$. The authors concluded that exenatide along with the changes in lifestyle resulted in substantial loss of weight, helped to regulate glucose, and resulted in reduced blood pressure as opposed to placebo plus changes in lifestyle [14].

Similarly, Bergenstal et al conducted a study on diabetic individuals in 2010 and followed them for 26 weeks after randomizing them to either exenatide or sitagliptin or pioglitazone [15]. The authors found more weight reduction among study subjects who received exenatide $(-2.3 \mathrm{~kg}, 95 \%$ confidence interval (CI): -2.9 to -1.7$)$ vs. sitagliptin $(-1.5 \mathrm{~kg}, 95 \% \mathrm{CI}:-2.4$ to $-0.7, \mathrm{P}=0.0002)$ or pioglitazone $(-5.1 \mathrm{~kg}, 95 \% \mathrm{CI}:-5.9$ to -4.3 , $\mathrm{P}<0.0001)$. Exenatide resulted in more weight reduction than other medications without causing hypoglycemia [15].

Two years later, Astrup et al conducted another study among obese individuals and followed them for 20 weeks [16]. There was $5.8 \mathrm{~kg}(95 \%$ CI: 3.7 - 8.0) more weight lost in group 1 as opposed to placebo and there was $3.0 \mathrm{~kg}$ additional weight loss as opposed to orlistat $(\mathrm{P}<0.001))$. There was 20 -week body fat reduced by 15.4 among those who took liraglutide and lean tissue by $2.0 \%$ (Table 4 ). The authors found that liraglutide is tolerated very well and there is sustainable weight loss over the period of 2 years, and there is also improvement in the risk of cardiovascular diseases [16]. Chun-Jun Li et al in 2014 followed obese and diabetic individuals after randomizing them to liraglutide [17]. It was found that subjects treated with liraglutide resulted in a mean weight reduction of 5.03 $\mathrm{kg}$ and $61.3 \%$ of the patients had a reduction of more than $5 \%$ of body weight as opposed to baseline [17]. The authors found that the body weight, waist circumference, total fat, lean mass, and fat percentage were substantially decreased when compared to baseline [17]. Another research done by Perna et al in 2016 assessed the effect of liraglutide on weight loss by following the study participants for 15 weeks [18]. A reduction was observed in the mean BMI $\left(-0.78 \mathrm{~kg} / \mathrm{m}^{2}\right)$, weight $(-2 \mathrm{~kg})$, fat mass $(-1.5 \mathrm{~kg})$ and android fat $(-0.9 \%)$ when compared to baseline, which revealed that treatment with liraglutide led to reduction in the mass of fat and android fat [18]. Another study conducted by Rondanelli et al on obese and diabetic individuals where they followed participants for 24 weeks. Af- 


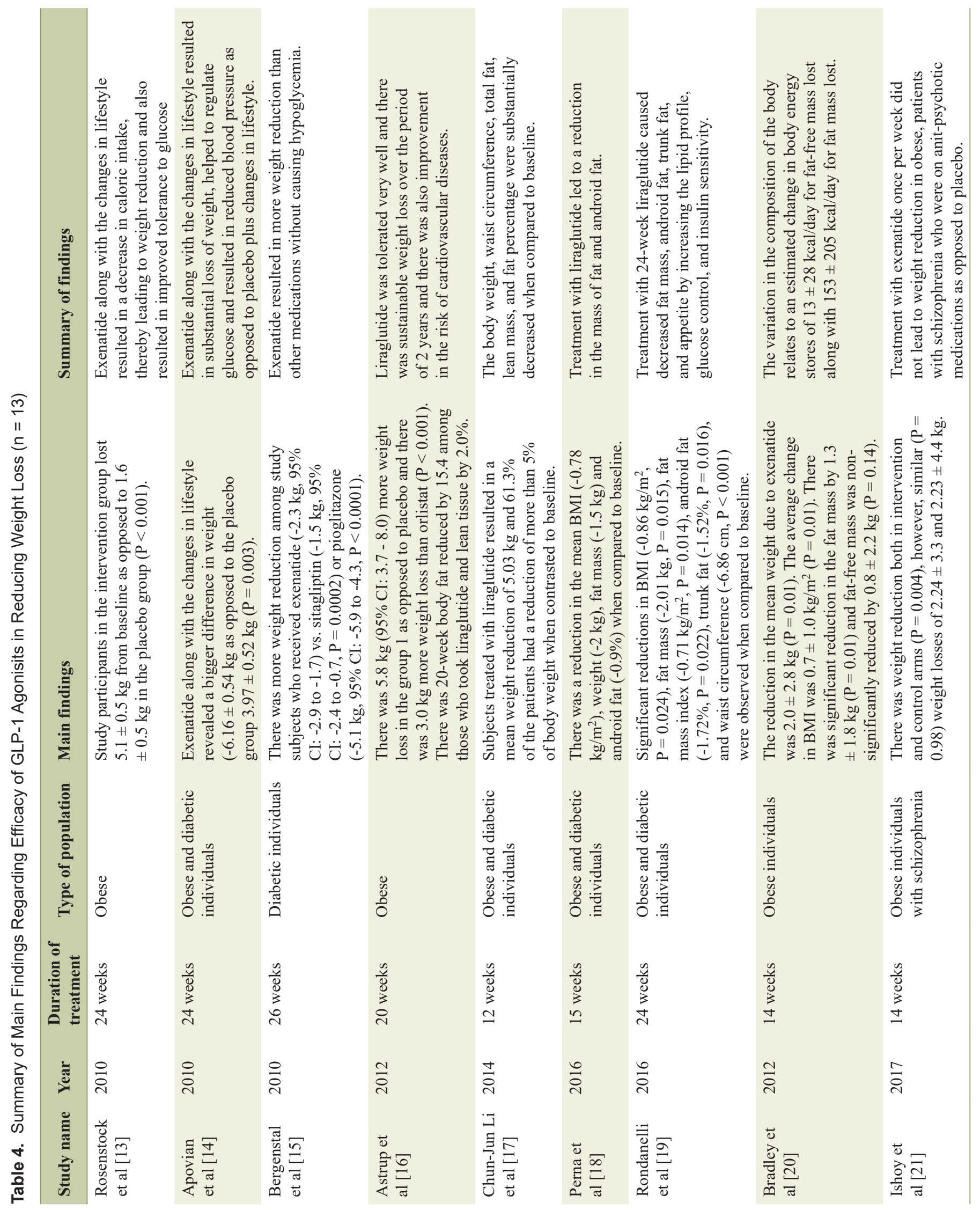




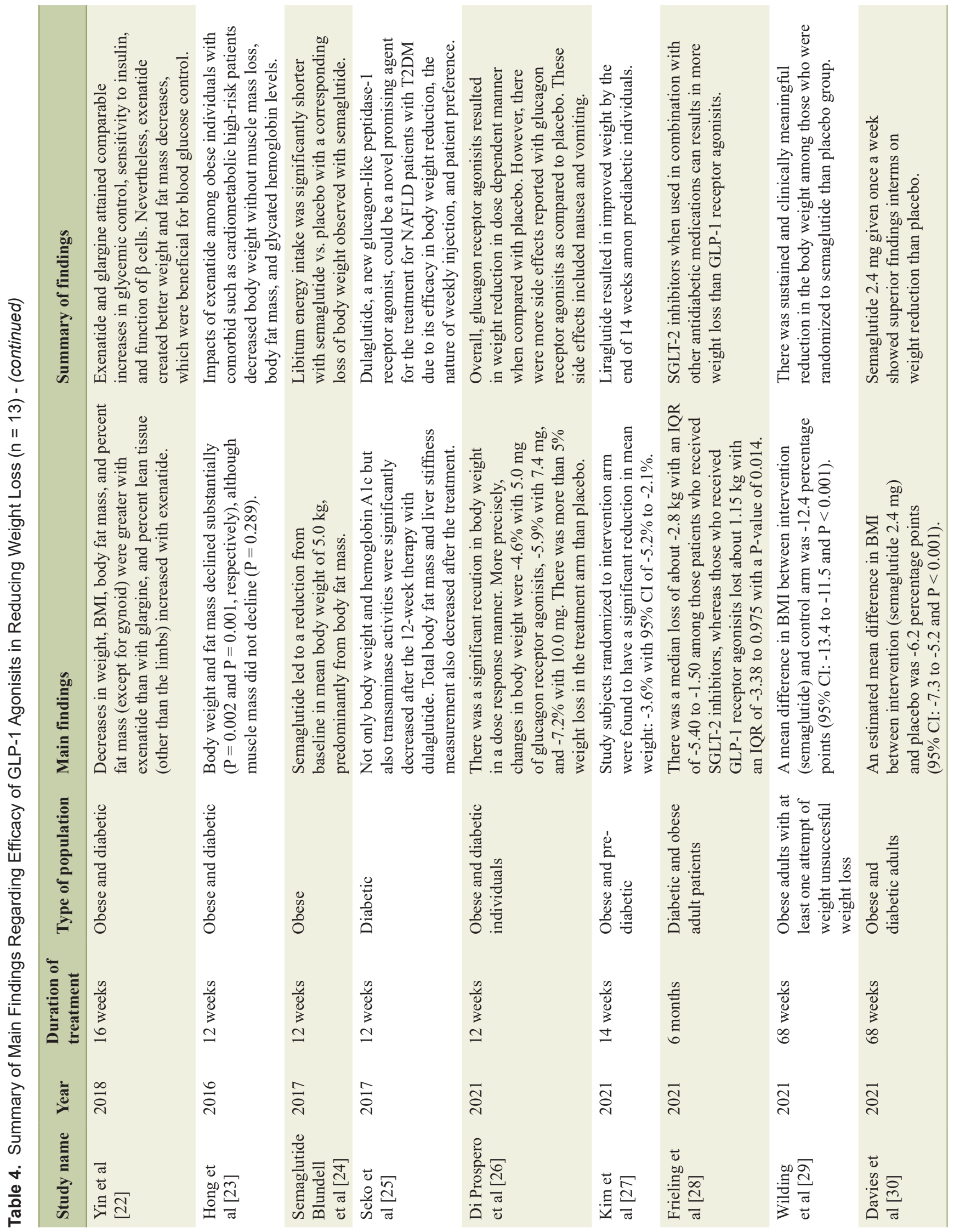




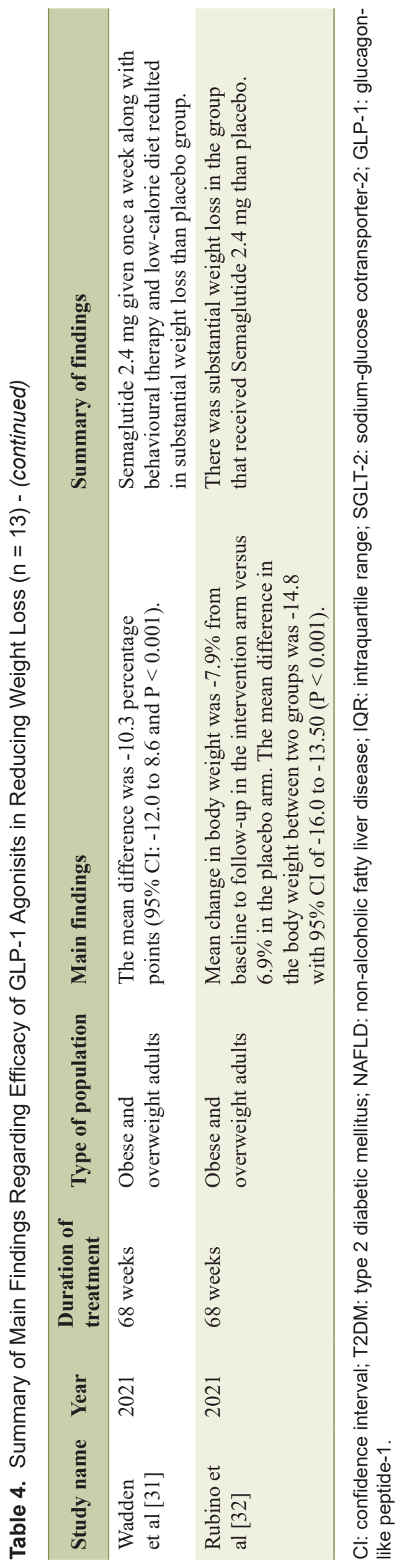

ter treating patients with liraglutide, significant reductions in BMI $\left(-0.86 \mathrm{~kg} / \mathrm{m}^{2}, \mathrm{P}=0.024\right)$, fat mass $(-2.01 \mathrm{~kg}, \mathrm{P}=0.015)$, fat mass index $\left(-0.71 \mathrm{~kg} / \mathrm{m}^{2}, \mathrm{P}=0.014\right)$, fat in android area $(-1.72 \%, \mathrm{P}=0.022)$, fat in trunk region $(-1.52 \%, \mathrm{P}=0.016)$, and waist circumference $(-6.86 \mathrm{~cm}, \mathrm{P}<0.001)$ were observed when compared to baseline [19].

Also, Bradley et al found a reduction in the mean weight of $2.0 \pm 2.8 \mathrm{~kg}(\mathrm{P}=0.01)$ due to exenatide. The mean difference in BMI was $0.7 \pm 1.0 \mathrm{~kg} / \mathrm{m}^{2}(\mathrm{P}=0.01)$. A substantial decrease was detected in the fat mass by $1.3 \pm 1.8 \mathrm{~kg}(\mathrm{P}=0.01)$ and fat-free mass was non-significantly reduced by $0.8 \pm 2.2$ $\mathrm{kg}(\mathrm{P}=0.14)[20]$. However, these findings were not supported by a study conducted by Ishoy et al in 2017 where authors found a weight reduction both in the intervention and control arms $(\mathrm{P}=0.004)$, though comparable $(\mathrm{P}=0.98)$, weight losses of $2.24 \pm 3.3$ and $2.23 \pm 4.4 \mathrm{~kg}$ were observed in both groups [21]. The authors observed that in contrast to placebo, treatment with exenatide once per week did not stimulate weight reduction among obese individuals, patients with schizophrenia who were on antipsychotic medications. In contrast, Yin et al conducted the study in 2018 by following obese and diabetic individuals for 16 weeks [22]. The authors found decreases in weight, BMI, body fat mass, and percent fat mass (except for gynoid) and such loss in weight was greater with exenatide than with glargine. Finally, Hong et al [23], Semaglutide Blundell et al [24], and Seko et al [25] found a reduction in the weight with exenatide, semaglutide, and dulaglutide, respectively.

Recently an RCT was conducted on 195 individuals who were randomized to either NJ-64565111 with three different doses of 5.0, 7.4, and $10.0 \mathrm{mg}$ and placebo group [26]. These participants were $18-70$ years old with a BMI of $35-50 \mathrm{~kg} / \mathrm{m}^{2}$ and were followed for 12 weeks after being assessed at baseline for various clinical and demographic factors. The study findings revealed a significant recution in body weight in a dose response manner [26]. More precisely, changes in body weight were $-4.6 \%$ with $5.0 \mathrm{mg}$ of glucagon receptor agonisits, $-5.9 \%$ with $7.4 \mathrm{mg}$, and $-7.2 \%$ with $10.0 \mathrm{mg}$ [26]. There was more than $5 \%$ weight loss in the treatment arm than placebo. Overall, glucagon receptor agonisits resulted in weight reduction in dose-dependent manner when compared with placebo. However, there were more side effects reported with glucagon receptor agonisits as compared to placebo. These side effects included nausea and vomiting [26]. Likewsie, another trial conducted by Kim et al on 35 patients for 14 weeks found the similar results in terms of weight loss [27]. More precisely, authors found that subjects randomized to intervention arm (liraglutide $1.8 \mathrm{mg} /$ day) were found to have a significant reduction in mean weight: $-3.6 \%$ with $95 \% \mathrm{CI}$ of $-5.2 \%$ to $-2.1 \%$ when compared to the placebo [27].

Further, one retrospective study conducted on 73 patients in 2021 for about 6 months on diabetic and obese individuals found a median loss of about $-2.8 \mathrm{~kg}$ with an intraquartile range (IQR) of -5.40 to -1.50 among those patients who received SGLT-2 inhibitors, whereas those who received GLP1Rass lost about $1.15 \mathrm{~kg}$ with an IQR of -3.38 to 0.975 with a P-value of 0.014 [28]. Auhtors concluded that SGLT-2 inhibitors when used in combination with other antidiabetic medications can result in more weight loss than GLP-1Ras [28]. 
We also assessed the findingds of STEP 1 to STEP 4 trials recently conducted in 2021. A STEP 1 study was a doubleblinded RCT of 1,961 participants conducted by Wilding et al. The authors found that a mean change in the BMI was $-14.9 \%$ in the group that was randomized to semaglutide when compared with $-2.4 \%$ change in the BMI among those who were randomized to placebo group [29]. Overall, the mean difference in BMI between intervention (semaglutide) and control arm was -12.4 percentage points $(95 \% \mathrm{CI}:-13.4$ to -11.5 and $\mathrm{P}$ $<0.001$ ) [29]. Further, change in the weight of study subjects in the intervention arm was -15.3 from baseline to follow-up in the intervention arm (semaglutide) compared to $-2.6 \mathrm{~kg}$ in the control arm with a mean difference of $-12.7 \mathrm{~kg}(95 \% \mathrm{CI}$ : -13.7 to -11.7$)$.

Likwise, a STEP 2 study group conducted a double-blinded RCT to assess the efficiacy of semaglutide $2.4 \mathrm{mg}$ versus $1.0 \mathrm{mg}$ and placebo for 68 weeks [30]. Authors found that there was an estimated change in mean body weight, from baseline to 68 weeks, of $-9.6 \%$ with the intervention arm when compared to $-3.4 \%$ with the placebo group. An estimated mean difference in BMI between intervention (semaglutide $2.4 \mathrm{mg}$ ) and placebo was -6.2 percentage points ( $95 \%$ CI: -7.3 to -5.2 and $\mathrm{P}<0.001$ ) [30]. There was a weight reduction of at least $5 \%$ among $68.8 \%$ of the study particopants in the semaglutide $2.4 \mathrm{mg}$ group when compared to $28.5 \%$ among placebo arm ( $\mathrm{P}$ $<0.0001$ ) [30].

Similarly a STEP 3 trial was conducted by Wadden et al in 2021 at 41 sites in the USA to compare the efficacy of semaglutide $2.4 \mathrm{mg}$ against placebo [31]. At the end of follow-up of 68 weeks, the estimated mean weight change was $-16.0 \%$ from baseline among those who were randomized to semaglutide $2.4 \mathrm{mg}$ when compared to $-5.7 \%$ for placebo group [31]. The mean difference was -10.3 percentage points $(95 \% \mathrm{CI}$ : -12.0 to 8.6 and $\mathrm{P}<0.001$ ). Around $87 \%$ of the study participants lost at least $5 \%$ of body weight in intervention arm versus $47.6 \%$ who lost the same percentage of body weight in placebo group $(\mathrm{P}<0.001)$ [31].

STEP 4 invetigators recently published findings of an RCT that compared the efficacy of semaglutide $2.4 \mathrm{mg}$ (once weekly) against placebo [32]. This trial was completed by 803 overweight and obese study participants for 68 weeks. The findings revealed that mean change in body weight was $-7.9 \%$ from baseline to follow-up in the intervention arm versus $6.9 \%$ in the placebo arm. The mean difference in the body weight between two groups was -14.8 with $95 \% \mathrm{CI}$ of -16.0 to -13.50 $(\mathrm{P}<0.001)[32]$.

\section{Discussion}

We undertook this systematic review to assess the efficacy of GLP-1 agonists to reduce the weight among obese diabetic or non-diabetic individuals. We reviewed all RCTs and case series that had assessed the efficacy of GLP-1 agonists such as exenatide, liraglutide, semaglutide, and dulaglutide and assessed the effect of these modalities on range of outcomes related to the weight. Overall, we found positive findings regarding these methods with equivalent results using different types of GLP-1 agonists. The findings of this systematic review revealed that in most cases, the weight reduction due to GLP1RAs was remarkable. GLP-1 receptors are found all over the human body, and therefore are expected to facilitate various physiological outcomes other than the glycemic control such as reduction in weight [33].

Our findings are consistent with the existing literature which previously have endorsed that apart from improving the glycemic levels, GLP-1RAs have been used by clinicians for the obesity as they can show promising results in reducing the weight, BMI and other constructs related to the obesity regardless of T2DM [34, 35]. For example, findings from a metaanalysis revealed that infusion of GLP-1 agonists resulted in an average of $\%$ of the libitum intake of energy when compared to the saline [36]. The underlying process by which GLP-1RAs help reduce the weight loss is not yet completely recognized. However, there is an evidence supporting that GLP-1RAs such as liraglutide raised satiety after meals, decreased appetite, reduced the consumption of food, and decreased energy expenditure [37]. Further, there is evidence that GLP-1RAs might postpone gastric emptying by inhibiting the vagal stimulation and in fact, it reduces weight loss by both working through peripheral and central pathways [36-38]. Hence, the existing premise endorses decreased hunger and intake of food, with no raised expenditure in energy, as the process causing weight loss associated with GLP-1RAs such as liraglutide. According to the studies related to the body, a reduction in the weight associated with liraglutide appears to parallel to a decrease in primarily visceral and subcutaneous fat instead of lean tissue mass [16]. There is also evidence of the analogous effects of liraglutide and exenatide as both result in remarkable suppression of food consumption and weight loss both among animals and human beings [38]. However, there is a need for more research about how the weight loss effect of liraglutide contrasts to that of exenatide.

Further the efficacy of GLP-1RAs can be linked to cardiovascular outcome trials (CVOTs) where there is evidence that CVOTs of GLP-1RAs among patients with T2DM have revealed that some of the GLP-1RAs have potential to reduce cardiovascular risk and may help to design and implement CVOTs in obesity in near future. Since obesity is one of the risk factors for cardiovascular morbidity and mortality, GLP-1RAs can be beneficial in reducing the risk of cardiovascular risk indirectly by reducing the weight of obese individuals. There is well-established evidence that weight reduction can lead to reduction in proinflammatory markers, which, in turn, can be helpful to improve the risk factors of coronary heart disease by reducing inflammation, thereby better cardiovascular outcomes.

\section{Strengths and limitations}

This review has endorsed the findings regarding the efficacy of GLP-1 agonists to help reduce the weight among obese individuals. The systematic review is strengthened due to robust evidence from both observational studies and RCTs, which is considered as the superior and gold standard in the hierarchy of study designs. We also found diverse studies from across the 
globe that gave us confidence that the GLP-1 agonists available to treat obesity can be generalized outside a given setting mainly across the globe. We found a considerable consistency in the primary outcomes for included studies as most of the studies assessed identical outcomes. However, the length of follow-up varied across the studies with lengthier follow-up for about 1 year, which might miss the recurrence that occurs in the longer run. Lastly, we tried to compare all modalities, which allude to understand the differences between different types of GLP-1 agonists to assess whether one is superior to the other.

\section{Conclusion}

Given their likely advantages further than glycemic control in reducing the weight, GLP-1 agonists may contribute to the treatment of obesity either among diabetic or non-diabetic individuals soon. Though, further research studies mainly large clinical trials are required to broaden and completely explain the favorable effects and potential side effects of GLP-1 agonists. Although this systematic review found positive effects of GLP-1 agonists in weight reduction, physicians need to write the prescriptions vigilantly to evade possibly side effects of the GLP-1 agonists, while offering opportunity for the overall health of obese individuals with or without diabetes.

\section{Acknowledgments}

None to declare.

\section{Financial Disclosure}

None to declare.

\section{Conflict of Interest}

None to declare.

\section{Data Availability}

The author declares that data supporting the findings of this study are available within the article.

\section{References}

1. Wu H, Ballantyne CM. Metabolic inflammation and insulin resistance in obesity. Circ Res. 2020;126(11):15491564.

2. Hamjane N, Benyahya F, Nourouti NG, Mechita MB, Barakat A. Cardiovascular diseases and metabolic abnormalities associated with obesity: What is the role of inflammatory responses? A systematic review. Microvasc Res.
2020;131:104023.

3. World Health Organization. Obesity and overweight. 2011. Available at: www.who.int/mediacentre/factsheets.

4. Shin SJ. Glucagon-like peptide-1 receptor agonists and their effects on weight reduction. J Diabetes Investig. 2012;3(6):490-491.

5. Eeg-Olofsson K, Cederholm J, Nilsson PM, Zethelius B, Nunez L, Gudbjornsdottir S, Eliasson B. Risk of cardiovascular disease and mortality in overweight and obese patients with type 2 diabetes: an observational study in 13,087 patients. Diabetologia. 2009;52(1):65-73.

6. Bailey C, Skouteris H, Teede H, Hill B, De Courten B, Walker R, Liew D, et al. Are lifestyle interventions to reduce excessive gestational weight gain cost effective? A systematic review. Curr Diab Rep. 2020;20(2):6.

7. Maula A, Kai J, Woolley AK, Weng S, Dhalwani N, Griffiths FE, Khunti K, et al. Educational weight loss interventions in obese and overweight adults with type 2 diabetes: a systematic review and meta-analysis of randomized controlled trials. Diabet Med. 2020;37(4):623-635.

8. Doyle ME, Egan JM. Mechanisms of action of glucagon-like peptide 1 in the pancreas. Pharmacol Ther. 2007;113(3):546-593.

9. Holst JJ. The physiology of glucagon-like peptide 1. Physiol Rev. 2007;87(4):1409-1439.

10. Verges B, Bonnard C, Renard E. Beyond glucose lowering: glucagon-like peptide-1 receptor agonists, body weight and the cardiovascular system. Diabetes Metab. 2011;37(6):477-488.

11. Kanoski SE, Rupprecht LE, Fortin SM, De Jonghe BC, Hayes MR. The role of nausea in food intake and body weight suppression by peripheral GLP-1 receptor agonists, exendin-4 and liraglutide. Neuropharmacology. 2012;62(56):1916-1927.

12. Moher D, Liberati A, Tetzlaff J, Altman DG, The PRISMA Group. Preferred reporting items for systematic reviews and meta-analyses: the PRISMA statement. Int J Surg. 2010;8(5):336-341.

13. Rosenstock J, Klaff LJ, Schwartz S, Northrup J, Holcombe $\mathrm{JH}$, Wilhelm K, Trautmann M. Effects of exenatide and lifestyle modification on body weight and glucose tolerance in obese subjects with and without pre-diabetes. Diabetes Care. 2010;33(6):1173-1175.

14. Apovian CM, Bergenstal RM, Cuddihy RM, Qu Y, Lenox S, Lewis MS, Glass LC. Effects of exenatide combined with lifestyle modification in patients with type 2 diabetes. Am J Med. 2010;123(5):468 e469-417.

15. Bergenstal RM, Wysham C, Macconell L, Malloy J, Walsh B, Yan P, Wilhelm K, et al. Efficacy and safety of exenatide once weekly versus sitagliptin or pioglitazone as an adjunct to metformin for treatment of type 2 diabetes (DURATION-2): a randomised trial. Lancet. 2010;376(9739):431439.

16. Astrup A, Carraro R, Finer N, Harper A, Kunesova M, Lean ME, Niskanen L, et al. Safety, tolerability and sustained weight loss over 2 years with the once-daily human GLP1 analog, liraglutide. Int J Obes (Lond). 2012;36(6):843854.

17. Li CJ, Yu Q, Yu P, Yu TL, Zhang QM, Lu S, Yu DM. 
Changes in liraglutide-induced body composition are related to modifications in plasma cardiac natriuretic peptides levels in obese type 2 diabetic patients. Cardiovasc Diabetol. 2014;13:36.

18. Perna S, Guido D, Bologna C, Solerte SB, Guerriero F, Isu A, Rondanelli M. Liraglutide and obesity in elderly: efficacy in fat loss and safety in order to prevent sarcopenia. A perspective case series study. Aging Clin Exp Res. 2016;28(6):1251-1257.

19. Rondanelli M, Perna S, Astrone P, Grugnetti A, Solerte SB, Guido D. Twenty-four-week effects of liraglutide on body composition, adherence to appetite, and lipid profile in overweight and obese patients with type 2 diabetes mellitus. Patient Prefer Adherence. 2016;10:407-413.

20. Bradley DP, Kulstad R, Racine N, Shenker Y, Meredith $\mathrm{M}$, Schoeller DA. Alterations in energy balance following exenatide administration. Appl Physiol Nutr Metab. 2012;37(5):893-899.

21. Ishoy PL, Knop FK, Broberg BV, Bak N, Andersen UB, Jorgensen NR, Holst JJ, et al. Effect of GLP-1 receptor agonist treatment on body weight in obese antipsychotictreated patients with schizophrenia: a randomized, placebo-controlled trial. Diabetes Obes Metab. 2017;19(2):162171.

22. Yin TT, Bi Y, Li P, Shen SM, Wang WM, Jiang C, Gao $\mathrm{CX}$, et al. Effects of exenatide versus insulin glargine on body composition in overweight and obese T2DM patients: a randomized controlled trial. Nutr Metab (Lond). 2018;15:67.

23. Hong JY, Park KY, Kim BJ, Hwang WM, Kim DH, Lim DM. Effects of short-term exenatide treatment on regional fat distribution, glycated hemoglobin levels, and aortic pulse wave velocity of obese type 2 diabetes mellitus patients. Endocrinol Metab (Seoul). 2016;31(1):80-85.

24. Blundell J, Finlayson G, Axelsen M, Flint A, Gibbons C, Kvist T, Hjerpsted JB. Effects of once-weekly semaglutide on appetite, energy intake, control of eating, food preference and body weight in subjects with obesity. Diabetes Obes Metab. 2017;19(9):1242-1251.

25. Seko Y, Sumida Y, Tanaka S, Mori K, Taketani H, Ishiba H, Hara T, et al. Effect of 12-week dulaglutide therapy in Japanese patients with biopsy-proven non-alcoholic fatty liver disease and type 2 diabetes mellitus. Hepatol Res. 2017;47(11):1206-1211.

26. Di Prospero NA, Yee J, Frustaci ME, Samtani MN, Alba M, Fleck P. Efficacy and safety of glucagon-like peptide-1/ glucagon receptor co-agonist JNJ-64565111 in individuals with type 2 diabetes mellitus and obesity: A randomized dose-ranging study. Clin Obes. 2021;11(2):e12433.

27. Kim SH, Abbasi F, Nachmanoff C, Stefanakis K, Kumar A, Kalra B, Savjani G, et al. Effect of the glucagon-like peptide-1 analogue liraglutide versus placebo treatment on circulating proglucagon-derived peptides that mediate improvements in body weight, insulin secretion and action: A randomized controlled trial. Diabetes Obes Metab. 2021;23(2):489-498.

28. Frieling K, Monte SV, Jacobs D, Albanese NP. Weight loss differences seen between glucagon-like peptide-1 receptor agonists and sodium-glucose cotransporter-2 inhibitors for treatment of type 2 diabetes. J Am Pharm Assoc (2003). 2021;:S1544-3191(21):00283-1.

29. Wilding JPH, Batterham RL, Calanna S, Davies M, Van Gaal LF, Lingvay I, McGowan BM, et al. Once-weekly semaglutide in adults with overweight or obesity. N Engl J Med. 2021;384(11):989.

30. Davies M, Faerch L, Jeppesen OK, Pakseresht A, Pedersen SD, Perreault L, Rosenstock J, et al. Semaglutide $2.4 \mathrm{mg}$ once a week in adults with overweight or obesity, and type 2 diabetes (STEP 2): a randomised, double-blind, double-dummy, placebo-controlled, phase 3 trial. Lancet. 2021;397(10278):971-984.

31. Wadden TA, Bailey TS, Billings LK, Davies M, Frias JP, Koroleva A, Lingvay I, et al. Effect of subcutaneous semaglutide vs placebo as an adjunct to intensive behavioral therapy on body weight in adults with overweight or obesity: the STEP 3 randomized clinical trial. JAMA. 2021;325(14):1403-1413.

32. Rubino D, Abrahamsson N, Davies M, Hesse D, Greenway FL, Jensen C, Lingvay I, et al. Effect of continued weekly subcutaneous semaglutide vs placebo on weight loss maintenance in adults with overweight or obesity: the STEP 4 randomized clinical trial. JAMA. 2021;325(14):14141425.

33. Kieffer TJ, Habener JF. The glucagon-like peptides. Endocr Rev. 1999;20(6):876-913.

34. Vilsboll T, Christensen M, Junker AE, Knop FK, Gluud LL. Effects of glucagon-like peptide-1 receptor agonists on weight loss: systematic review and meta-analyses of randomised controlled trials. BMJ. 2012;344:d7771.

35. Zhang F, Tong Y, Su N, Li Y, Tang L, Huang L, Tong N. Weight loss effect of glucagon-like peptide-1 mimetics on obese/overweight adults without diabetes: A systematic review and meta-analysis of randomized controlled trials. J Diabetes. 2015;7(3):329-339.

36. Verdich C, Flint A, Gutzwiller JP, Naslund E, Beglinger C, Hellstrom PM, Long SJ, et al. A meta-analysis of the effect of glucagon-like peptide-1 (7-36) amide on ad libitum energy intake in humans. J Clin Endocrinol Metab. 2001;86(9):4382-4389.

37. van Bloemendaal L, Ten Kulve JS, la Fleur SE, Ijzerman RG, Diamant M. Effects of glucagon-like peptide 1 on appetite and body weight: focus on the CNS. J Endocrinol. 2014;221(1):T1-16.

38. Madsbad S. The role of glucagon-like peptide-1 impairment in obesity and potential therapeutic implications. Diabetes Obes Metab. 2014;16(1):9-21. 